\title{
Directed migration of circulating polymorphonuclear leucocytes in patients with rheumatoid arthritis: a defect in the plasma
}

\author{
J. R. WALKER, D. W. JAMES, AND M. J. H. SMITH \\ From the Biochemical Pharmacology Research Unit, Department of Chemical Pathology, King's College \\ Hospital Medical School, Denmark Hill, London SE5 8 RX
}

SUMMARY The migration of peripheral polymorphonuclear leucocytes (PMNs) of patients with rheumatoid arthritis has been studied both in vivo and in vitro. A significant reduction in the accumulation of PMNs in skin chambers in patients with rheumatoid arthritis compared to controls was observed but no defect in cell movement was detected when the isolated PMNs from the patients were exposed to activated control plasma. However, when PMNs from the control group were tested against activated plasma from patients with rheumatoid arthritis there was a significant decrease in their chemotactic response. It is proposed that there is a humoral defect in the plasma of the patients.

Abnormalities of polymorphonuclear leucocyte (PMN) migration have been described in many diseases (Quie and Cates, 1977). Evidence has been presented for a defect in the in vitro chemotactic responsiveness of the cells in patients with rheumatoid arthritis (Mowat and Baum, 1971). It was suggested that ingestion of immune complexes may be a possible explanation for the reduced responsiveness of the PMNs to chemotaxins. More recent work with patients with rheumatoid arthritis (Roberts-Thomson et al., 1976) and Felty's syndrome (Hurd et al., 1977) has lent support to the proposal. Other workers (Goetzl, 1976), however, have been unable either to confirm the abnormal movement in vitro by PMNs from rheumatoid patients reported in the earlier studies or to demonstrate an abnormality in the accumulation of PMNs into skin chambers in similar patients (Segal and Loewi, 1976).

In this communication we describe the findings from 2 separate series of experiments which were designed to re-examine the chemotactic response of PMNs in rheumatoid arthritis both in vivo, using skin chambers to observe the accumulation of PMNs into autologous serum, and in vitro using modified Boyden chambers to measure the directed migration of cells in both rheumatoid and control groups. The experiments were designed to determine if a defect in cell movement existed in patients with

Accepted for publication 25 July 1978.

Correspondence to Dr J. R. Walker. rheumatoid arthritis and, if so, whether the defect was associated with either the leucocytes themselves or with some abnormality of the plasma.

\section{Patients and methods}

All the patients reported on in these studies were attending rheumatology outpatient clinics at King's College Hospital and had definite or classical rheumatoid arthritis (American Rheumatism Association criteria).

In the in vitro studies 28 patients constituted the rheumatoid group (12 male and 16 female; average age 58.3 years, range $34-76$ ) with a mean duration of disease of 7 years (range 1-19 years). These patients were on a variety of drug treatments including gold, penicillamine, and steroids, and the majority were also taking a nonsteroidal antiinflammatory drug such as indomethacin or salicylate. The control group consisted of 23 patients ( 9 male and 14 female; average age 51 years, range 23-76 years) suffering from a variety of noninflammatory diseases such as low back pain and osteoarthrosis. The majority of these patients were not receiving any drug therapy.

The in vivo studies were performed on 17 patients with rheumatoid arthritis (4 male and 13 female; average age $60 \cdot 1$ years, range $49-76$ years) with a mean duration of disease of 11.8 years (range 1-35 years). These patients were all being treated with nonsteroidal anti-inflammatory drugs. In addition 1 
patient was on gold treatment and 16 were on Dpenicillamine or fenclofenac or placebo as part of a double blind clinical trial. The control group consisted of 2 sub groups-one of 5 young healthy laboratory workers (average age 24 years, range 21-27 years), and the other of 11 older patients with osteoarthrosis (average age 53.1 years, range 43-64 years). Two of the patients in this second group were receiving nonsteroidal anti-inflammatory drugs.

\section{IN VITRO PROCEDURES}

Samples $(20 \mathrm{ml})$ of blood were obtained by venepuncture and transferred to bottles containing 200 units of preservative-free heparin. $5 \mathrm{ml}$ of each sample was centrifuged ( $15 \mathrm{~min}$ at $2000 \mathrm{~g}$ ), the plasma removed, and samples used as described below either immediately or after storage at $-20^{\circ} \mathrm{C}$ for no longer than 2 weeks. Cell populations were obtained from the remaining blood by dextran sedimentation as described previously (Walker et al., 1975). All cell populations $(75-80 \%$ PMNs) were resuspended to give a concentration of between 2.0 and $2.5 \times$ $10^{6}$ cells $\mathrm{ml}^{-1}$ in Medium 199 buffered to $\mathrm{pH} 7 \cdot 3$ with HEPES (25 mM). Chemotaxins were generated in the plasma samples by incubation with zymosan $\left(2 \mathrm{mg} \mathrm{ml}{ }^{-1}\right)$ for $30 \mathrm{~min}$ at $37^{\circ} \mathrm{C}$ followed by heat inactivation for $30 \mathrm{~min}$ at $56^{\circ} \mathrm{C}$. The zymosan was removed by centrifugation and the activated plasmas were diluted 1 in 10 with Medium 199. Directed migration was measured in modified Boyden chambers (Neuroprobe, Inc., Maryland, USA) fitted with $5 \mu \mathrm{m}$ Millipore filters. The cell suspension $(0.5 \mathrm{ml})$ was placed in the upper chamber and $1.5 \mathrm{ml}$ of the diluted plasma into the lower chamber, and each experiment was performed in duplicate. The chambers were incubated at $37^{\circ} \mathrm{C}$ for $2 \mathrm{~h}$, after which the filters were removed, fixed, and stained. The number of cells that had reached the lower surface of the filter was counted in 5 separate highpower fields under $400 \times$ magnification. $\alpha_{1}$-Antitrypsin, $\alpha_{2}$-macroglobulin, and total $\mathrm{C} 3$ and $\mathrm{C} 4$ concentrations in the plasma of the rheumatoid patients were determined with commercially available immunodiffusion plates (Hoechst Pharmaceuticals). Functional factor B and total alternative pathway capacity were measured by means of bystander haemolysis of guinea-pig erythrocytes suspended in agarose as the indicator system (Martin et al., 1976). Measurement of other plasma proteins such as immune complexes or cryoglobulins was not carried out.

IN VIVO PROCEDURES

A modification of the skin chamber technique described by Senn et al., (1969) was used. Blood was taken from each individual immediately before the $\frac{\bar{\rho}}{\rho}$. experiment from which cell-free serum was obtained. After prehydration of the skin with pads soaked in normal saline the skin over the flexor aspect of the forearm was cleaned with $70 \%$ isopropyl alcohol. Two small abrasions were made by means of an electric drill with a spherical dental burr $(0.6 \mathrm{~cm} \cong$ diameter). The mean area of the abrasions was $0.080 \pm$ SEM $0.005 \mathrm{~cm}^{2}, \mathrm{n}=50$. Occasional small ${ }^{\text {s }}$ bleeding points were seen, but bleeding stopped. within 3 minutes. Plastic skin chambers $(2.5 \mathrm{ml} \vec{\omega}$ capacity) were placed over the abrasions and were $\rho$ held in place by a silicone adhesive (Medical Adhesive B, Dow Corning Corporation) and sur- $\frac{0}{\omega}$ gical tape. Autologous serum $(2 \mathrm{ml})$ was intro- $\infty$ duced into each chamber through a small hole ${ }_{i}^{\omega}$ which was plugged. The chambers were kept on the $\vec{G}$ arm for $24 \mathrm{~h}$, after which the serum was aspirated 0 and placed into plastic tubes. The area of each abrasion was measured with a Vernier caliper after $\subset$ removal of the skin chamber. The number of leucocytes in the serum was determined by standard $\overrightarrow{0}$ methods and the number of cells migrating into $1 \mathrm{ml}$ of serum per $\mathrm{cm}^{2}$ in $24 \mathrm{~h}$ was calculated. Over $95 \%$. of the migrating cells were PMNs.

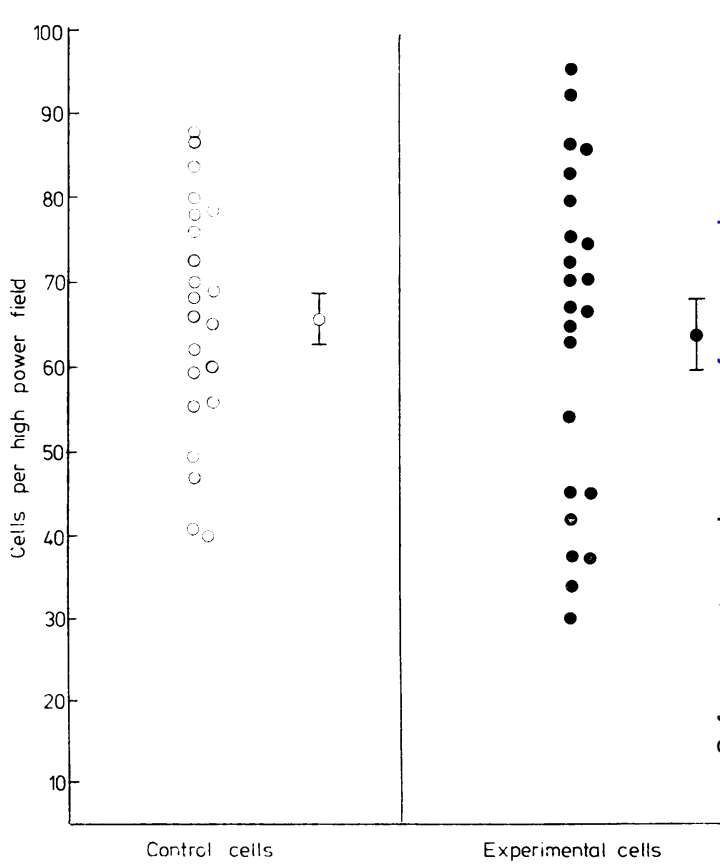

Fig. 1 Response of polymorphonuclear leucocytes from control and experimental groups to activated plasma from control group. O PMNs from control group. PPMN from experimental group. I Mean = standard error. 


\section{Results}

IN VITRO

The results in Fig. 1 show that there was no significant difference in the directed migration of the peripheral leucocytes prepared from either group of patients when the cells were exposed to activated control plasma in vitro. In contrast, the results in Fig. 2 show clearly that leucocytes prepared from control patients showed a significantly diminished response when exposed to activated rheumatoid plasma (41\% reduction, $\mathrm{P}<\mathbf{0} .001$, Student's $t$ test) when compared to activated control plasma. The response of control cells to control activated plasma did not vary more than $10 \%$ from day to day. This reduced chemotactic attraction bore no relation to other parameters measured, that is, duration and severity of the rheumatoid arthritis, erythrocyte sedimentation rate (ESR), the presence of rheumatoid factor, $\alpha_{1}$-antitrypsin and $\alpha_{2}$-macroglobulin concentrations nor to drug therapy. No deficiencies in the patients' plasma as regards the total concentrations of the complement components $\mathrm{C} 3$ and $\mathrm{C} 4$, the functional activity of Factor $B$, and the functional capacity of the alternative pathway were observed.

IN VIVO

Fig. 3 illustrates the results obtained with the

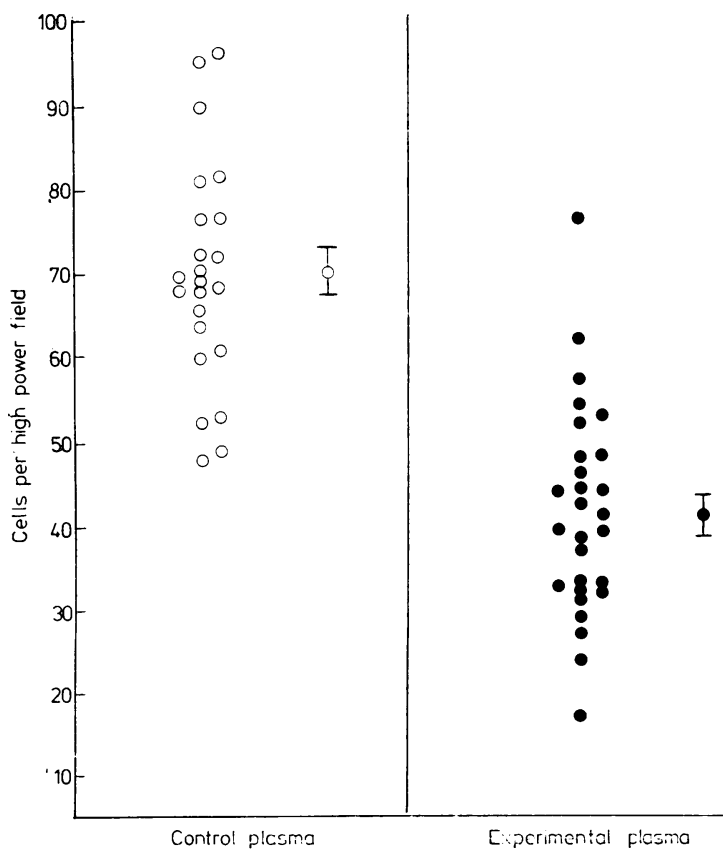

Fig. 2 Response of control polymorphonuclear leucocytes to individual activated plasma samples from control and experimental groups. Symbols as for Fig. 1

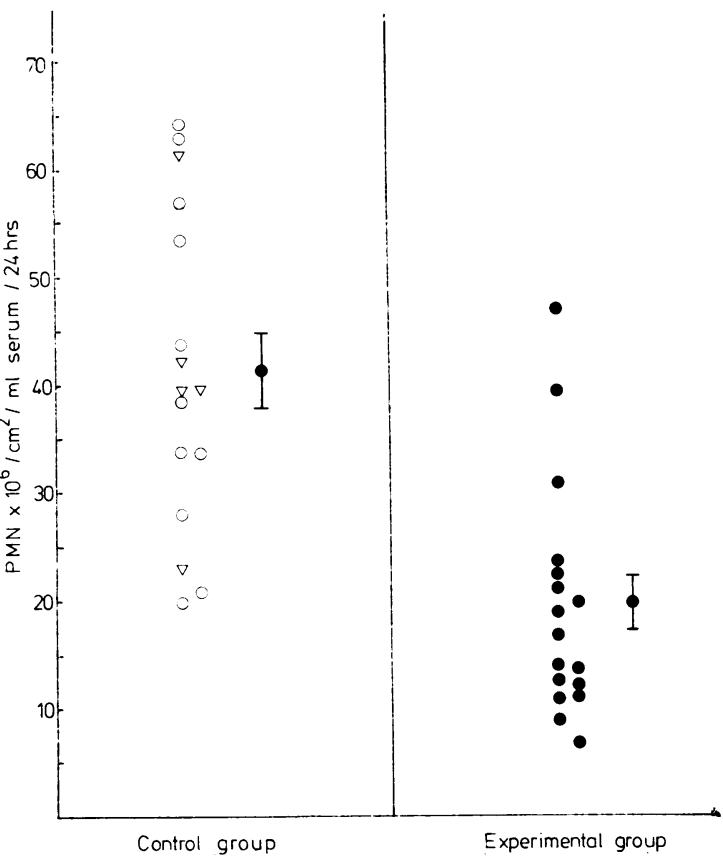

Fig. 3 Comparison of PMN accumulation in skin chambers between control and rheumatoid patients. O Age-matched controls. $\nabla$ 'Young' control group.

- Experimental group. $\Phi_{\mathbf{Q}}^{\top}$ Mean $=$ standard error

control subgroups compared with those obtained with the rheumatoid patients. There was no difference in cell accumulation between the two control subgroups, but there was a significant reduction in PMN accumulation in the patients with theumatoid arthritis $(46.5 \%, \quad P<0.001)$. This diminished cellular responsiveness in vivo was again unrelated to other parameters such as ESR, articular index, duration of disease, the patient's age, or to drug therapy.

\section{Discussion}

Various defects have been reported, either cellular or humoral, in several chronic inflammatory diseases including rheumatoid arthritis (Mowat and Baum, 1971), systemic lupus erythematosus (Clark et al., 1974; Goetzl, 1976) sarcoidosis (Maderazo et al., 1976; Gange et al., 1977) and Crohn's disease (Segal and Loewi, 1976).

The observation in the present paper of a diminished accumulation of PMNs in skin chambers in rheumatoid arthritis patients is contrary to other findings (Segal and Loewi, 1976) but reflects our in vitro observations. The accumulation of PMNs in 
vivo is the end product of a variety of effects such as directed and stimulated random migration, vascular status, blood flow, and the presence or absence of serum inhibitors. It is thus impossible to distinguish from the observations in vivo which of these many factors may be responsible for the depressed cellular response. However, none of the patients or controls in this or the subsequent study showed any indications of major differences in vascular status such as Raynaud's disease. Although the in vitro experiments were performed with cells and plasma samples obtained from a different series of patients, it has been possible to demonstrate that, while PMNs obtained from these patients respond normally when exposed to activated control plasma, there is a factor or factors present in their plasma which in some way depresses the chemotactic response of normal PMNs when exposed to their activated plasma in modified Boyden chambers.

Inhibition of the release of chemotaxins during complement activation by zymosan is unlikely to be a mode of action, since the measurement of alternative pathway capacity which utilised whole plasma was unaffected. A further mode of action of the plasma material may be the deactivation of chemotaxins once they have been generated from the parent complement molecules. Such materials have been described in a variety of diseases (Ward et al., 1977; Van Epps and Williams, 1978). Using an agarose plate chemotactic assay we have found that control PMNs migrate normally towards activated rheumatoid plasma (results not shown). The apparent discrepancy between the 2 methods indicates, firstly, that the factor(s) is exerting its inhibitory action directly on the cell (Goetzl, 1975), and, secondly, the material is of considerably higher molecular weight than the complementderived chemotactic peptides. Experiments to confirm the mode of action and to clarify the physical properties of the inhibitory materials are in progress.

A possible criticism of these results is that the drug therapies employed may exert an effect on the in vivo cell accumulation or in vitro migration studies. While this cannot be totally ruled out, there is no apparent correlation between the drug treatment of the patients who received skin chambers or the plasma activities of those patients used in the in vitro studies.

The presence of such an inhibitor raises a number of questions with respect to the disease. First, does it cause the propensity to infection observed in such patients? Secondly, is its presence a fundamental manifestation of the disease? A related and important query is whether diminished chemotactic responsiveness is a pathological reaction to the disease process or whether it represents a normal defence mechanism. The present experiments have been: concerned only with the behaviour of PMNs, and its is of some importance to extend the work to investio gate whether the responsiveness of mononuclearo cells, which are of more importance in chronics, inflammation, is also affected.

We wish to thank Dr E. B. D. Hamilton, Dr C. J. Goodwill, and Dr H. Berry for allowing us access to patients in theiro care. J. R. Walker is the holder of the Cowburn scholarship at King's College Hospital Medical School.

\section{References}

Clark, R. A., Kimball, H. R., and Decker, J. L. (1974) Neutrophil chemotaxis in systemic lupus erythematosus Annals of the Rheumatic Diseases, 33, 167-172.

Gange, R. W., Black, M. M., Carrington, P., and McKerron, R. (1977). Defective neutrophil migration in sarcoidosis. Lancet, 2, 379-381.

Goetzl, E. J. (1975). Plasma and cell-derived inhibitors of human neutrophil chemotaxis. Annals of the New York Academy of Sciences, 256, 210-220.

Goetzl, E. J. (1976). Defective responsiveness to ascorbico acid of neutrophil random and chemotactic migration in Felty's syndrome and systemiclupus erythematosus. Annals of the Rheumatic Diseases, 35, 510-515.

Hurd, E. R., Andreis, M., and Ziff, M. (1977). Phagocytosis of immune complexes by polymorphonuclear leucocytes in patients with Felty's syndrome. Clinical and Experi-O mental Immunology, 28, 413-425.

Maderazo, E. G., Ward, P. A., Woronick, C. L., Kubik, J., and DeGraff, A. C. (1976). Leucotactic dysfunction in sarcoidosis. Annals of Internal Medicine, 84, 414-419.

Martin, A., Lachmann, P. J., Halbwachs, L., and Hobart, M. J. (1976). Haemolytic diffusion plate assays for factors $B$ and $\mathbf{D}$ of the alternative pathway of complement activa-O tion. Immunochemistry, 13, 317-324.

Mowat, A. G., and Baum, J. (1971). Chemotaxis of polymorphonuclear leucocytes from patients with rheumatoid arthritis. Journal of Clinical Investigation, 50, 2541-2549.

Quie, P. G., and Cates, K. L. (1977). Clinical conditions. associated with defective polymorphonuclear leucocyteo chemotaxis. American Journal of Pathology, 88, 711-726.

Roberts-Thomson, P. J., Hazelman, B. L., Barnett, I. G.,o Maclennan, I. C. M., and Mowat, A. G. (1976). Factors relating to circulating immune complexes in rheumatoid arthritis. Annals of the Rheumatic Diseases, 35, 314-320. 극

Segal, A. W., and Loewi, G. (1976). Neutrophil dysfunction in Crohn's disease. Lancet, 2, 219-221.

Senn, H., Holland, J. F., and Baneriee, T. (1969). Kinetio and comparative studies on localised leucocyte mobilisation in normal man. Journal of Laboratory and Clinical Medicine, $\omega$ 74, 742-756.

Van Epps, D. E., and Williams, R. C. (1978). Serum inhibitors of leucocyte chemotaxis and their relationship to skin testC anergy. Leucocyte Chemotaxis, p. 237. Ed. J. I. Gallin and P. G. Quie. Raven Press: New York.

Walker, J. R., Badcock, J. K., Ford-Hutchinson, A. W., T Smith, M. J. H., and Billimoria, F. J. (1975). Effects of aO human plasma fraction on the release of chemotaction factors and anaphylatoxin from complement. Journal of Pharmacy and Pharmacology, 27, 747-753.

Ward, P. A., Kent, J. J., and Kreutzer, D. L. (1977). Regulatory dysfunction in leucotaxis. American Journal of Pathology, 88, 701-707. 